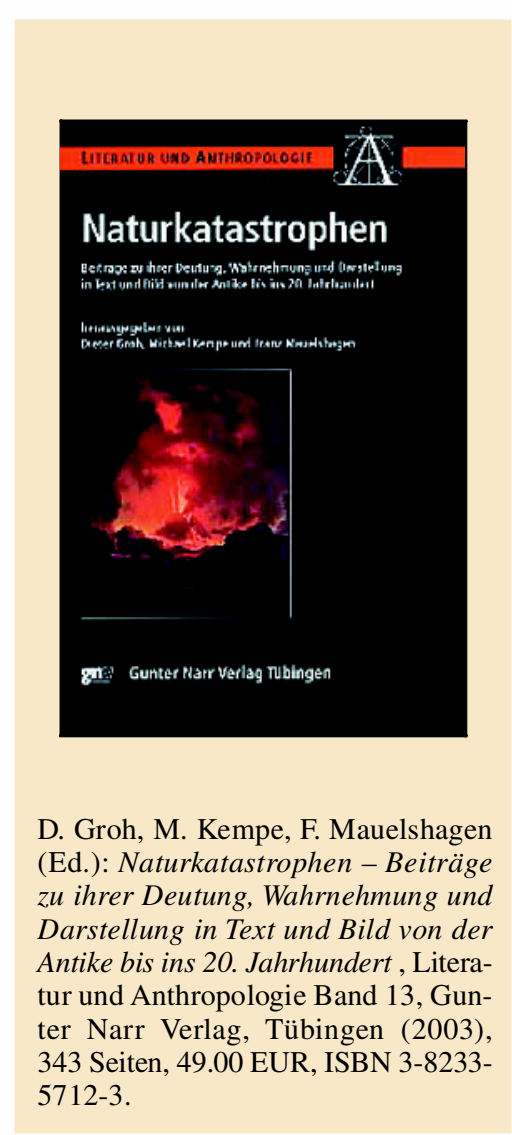

$\mathrm{D}$ en Autoren der Aufsätze - die rein maskuline Form muß verwendet werden, weil die 19 Beiträge ausschließlich aus Männer-Feder stammen - geht es in erster Linie darum, bereits eingetretene, also faktische und damit im engen Sinne "historische" Ereignisse mit für den Menschen verheerenden Folgen wahrzunehmen, darzustellen und zu deuten. Dieser anthropozentrische Zugang, der die extremen Naturereignisse im Sinne naturwissenschaftlicher Prozesse selbst ausblendet, ist im Verständnis der Autoren als Historiker begründet - er wird in dem Buch mit seinem historisch-sozialwissenschaftlichen sowie mit wahrneh-mungsorientierten Ansätzen fortgesetzt. In einzelnen Etappen von der Antike bis zur Gegenwart erfahren wir, wie Menschen Erdbeben, Vulkanausbrüche oder Hochwasser beobachteten, in Schrift und Bild dokumentierten und - nicht immer zweckfrei - zu deuten und zu erklären suchten. Die Reise geht von schriftlichen Quellen über Bildzeugnisse bis hin zum TV-basierten "Katastrophenvoyeurismus" (Beitrag Kirchmann). Interessant ist die Vielfalt verschiedener naturkundlicher bis metaphysischer Ansätze, die stets jeweilige gesellschaftspolitische und religiöse Rahmenbedingungen widerspiegeln.

\title{
Als Naturkatastrophen noch Naturkatastrophen waren
}

Naturkatastrophenforschung sei »gesellschaftsbezogen und wirkungsorientiert « und deshalb Teil der Sozial- und Geisteswissenschaft - so informiert uns die von den Herausgebern Groh, Kempe und Mauelshagen verfaßte Einleitung. Es handelt sich um eine Sammlung von Aufsätzen auf Basis von Vorträgen, die auf der Tagung "Naturkatastrophen und ihre Wahrnehmung in der Geschichte des Menschen" im November 2000 in Konstanz gehalten wurden. Die Herausgeber des Bandes setzen mit dieser Aussage einen wohltuend selbstbewußten Akzent und bringen, so anscheinend ihr Anspruch, Konkurrenz in ein Forschungsgebiet, das bislang von den Natur- und Ingenieurwissenschaften dominiert ist. Diese haben es erst ansatzweise geschaff, die Brücke zu den Sozial- und Geisteswissenschaften zu schlagen-aus der Sicht eines interdisziplinär Forschenden ist das zu bemängeln. Im Ergebnis zeigt sich aber mit dem vorliegenden Buch, daß auch die Sozial- und Geisteswissenschaftler diesen Mangel nicht zu beheben vermögen. Dabei bietet sich gerade wirkungsorientierte Forschung für interdisziplinäre Ansätze an.

Bei der Aufzeichnung der historischen Ereignisabläufe erfrischend wirken Ausführungen zum jeweils vorhandenen Stand des empirischen Wissens um natürliche Prozesse und zu naturwissenschaftlich fundierten Erklärungsversuchen. Definition und Verständnis von Naturkatastrophen haben sich allein in der zweiten Hälfte des zwanzigsten Jahrhunderts rasant gewandelt (Beitrag Engels). Diese Erkenntnis unterstreicht, wie wichtig es ist, die soziologisch-historische Dimension in der interdisziplinären Erforschung von Naturgefahren und -katastrophen zu berücksichtigen. Damit sind wir auch beim Begriff Naturkatastrophen. Dessen Inhalt und Bedeutung werden zum einen extensiv diskutiert (Beitrag Pfister). Zum anderen führen die Autoren aber gedankenlos auch wieder neue Begriffe in Form gräßlicher Wortschöpfungen wie "Naturhazards" (Beitrag Grob et al.) oder "Wolfskatastrophe" (Beitrag Siemer) ein. Das mag innovativ erscheinen, trägt aber letztlich wenig bei zur Förderung einer gemeinsamen Sprache und damit eines gemeinsamen Verständnisses über alle Wissenschaftszweige hinweg.

Mit Nachdruck weisen verschiedene Autoren darauf hin, daß sich in den letzten 20 Jahren das Verhältnis von Natur und Mensch grundlegend verändert hat und der Mensch von der »Rolle des Opfers mehr und mehr in die des Täters rückt « (Einleitung Groh et. al) und dabei die Natur in seiner »omnipräsenten und potenten Art zum Manipulationsopfer degradiert «(Beitrag Engels). Besonders der Praktiker mag diese Interpretation ähnlich einseitig oder vereinfachend emp- finden wie dies der straftheologischen Deutung früherer Jahrhunderte vorgeworfen werden kann. Danach waren $\mathrm{Na}$ turkatastrophen als Zeichen göttlichen Zorns über die Sündhaftigkeit des Menschen und somit als Strafgerichte zu deuten (Beitrag Jakubowski-Tiessen). Dabei interessierte offenbar die Deutung der "Botschaft der Götter" oft mehr als die eigentliche Katastrophe (Beitrag Meier) deuten sich hier Parallelen zu heutigen Forschungszweigen an? Interessant ist auch, daß nicht erst heute, sondern bereits zu Zeiten des Sonnenkönigs Ludwig XIV. Naturkatastrophen dazu benutzt wurden, politische Ziele zu verfolgen (Beitrag Strube). Die mittelalterlich-neuzeitlichen Deutungsversuche von Pest (Beitrag Bulst) oder Syphilis (Beitrag Walter) dürften eher medizinhistorisches denn katastrophenmanagementbezogenes Interesse hervorrufen. Reizvoll wäre hier gewesen, den Bogen zur heutigen Gesellschaft zu schlagen mit der Frage, wie diese mit neuzeitlichen Krankheitsbildern wie AIDS, BSE oder SARS umgeht.

Historische Quellen als Zeugnisse vergangener Naturkatastrophen sollten selbst bei scheinbar hoher Detailschärfe kritisch beleuchtet werden, vor allem mit Blick auf die persönliche Motivation des Autors sowie die großräumige gesellschaftliche und politische Situation (Propaganda, Zensur, Weltuntergangsstimmung et cetera). Obgleich Skizzen der frühen Geologie interessante Einblicke in die historische Formulierung wissenschaftlicher Katastrophentheorien liefern, läßt die teils subjektiv anmutende, kunstgeschichtliche Deutung von Bildzeugnissen (Beitrag Felfe) Zweifel aufkommen, ob die Quel- 
len tatsächlich mit der gebotenen kritischen Distanz betrachtet wurden. In diesem Sinne sind sie unzureichend und eben »keine Beweisaufnahmen von $\mathrm{Au}-$ genzeugen, vielmehr Phantasmagorien der entfesselten Elemente und Parabeln über die Ausweglosigkeit der Existenz« (Beitrag Weber). Die geschickt gewählte Reihenfolge der einzelnen Beiträge in der Aufsatzsammlung ermöglicht es den Lesenden, treffliche Parallelen zur sensationslüsternen und damit ebenfalls nicht manipulationsfreien Darstellung heutiger Naturkatastrophen in modernen Medien wie dem Fernsehen zu ziehen (Beitrag Kramer).

Interessante Lektüre ist auch der vierte Teil des Buches zum Thema "Integrationsprozesse". Die Autoren präsentieren pointiert Material zur gesellschaftlichen Solidarität bei der Bewältigung ausgesuchter Ereignisse wie historischer Bergstürze in den Schweizer Alpen (Beitrag Pfister) oder dem Oderhochwasser von 1997 (Beitrag Döring). Der letzte Teil des Buches, "Chaos und Ordnung" gewidmet, verspricht nichts Minderes: »Die Erde musste wieder einmal Samba tanzen«, heißt es in der Einleitung zu Haunschilds Beitrag "Erdbeben und das Ewige in Süditalien - Zur Geophysik der Macht", der sich wie der ethnologische Reisebericht eines Weltenbummlers liest. Der Beitrag verdeutlicht die beinahe grenzenlose Regenerationsfähigkeit der betroffenen Gesellschaft nach einer Naturkatastrophe. Ist es demnach gerechtfertigt, Naturkatastrophenforschung als »gesellschaftsbe- zogen und wirkungsorientiert « zu bezeichnen und sie deshalb für die Sozialund Geisteswissenschaften zu reklamieren, wie es die Herausgeber tun? Dem grundlegenden Anspruch des Sammelbandes nach erscheint Naturkatastrophenforschung zwar als auch wirkungsorientiert, aber vorrangig als quellenbezogen oder -bestimmt. Mehrere Autoren merken selbst an, es sei mitunter zweifelhaft, inwieweit historische Dokumente den gesellschaftlichen Umgang mit Naturkatastrophen adäquat zu beleuchten vermögen. Schließlich handelt es sich bei diesen Dokumenten doch stets um Blickwinkel eines oder mehrerer Individuen, deren Hauptmotiv nicht immer das der objektiven Dokumentation gewesen sein mag. Die Interpretation solcher Quellen erscheint spätestens dann etwas unkritisch, wenn eine gesellschaftliche Allgemeingültigkeit abgeleitet wird.

Die Lektüre des Buches ist allemal zu empfehlen. Die inhaltlich breit gefächerten und profund recherchierten Aufsätze eröffnen interessante Einblicke in den historischen (Be-)Deutungswandel von Naturkatastrophen. Spannend ist vor allem, wie die Gesellschaft in den unterschiedlichsten Kulturräumen über die Jahrhunderte mit Naturkatastrophen fertig geworden ist. Bedenkt man, daß die Gesellschaft in ihrer zunehmenden Verletzbarkeit gegenüber Naturkatastrophen künftig noch in viel stärkerem Ausmaß betroffen sein kann, behandelt das Buch Themen, wie sie aktueller nicht sein könnten. Der Praktiker vermißt aller- dings vor allem eine problembezogene Gesamtschau darauf, wie Gesellschaften Risiken wahrnehmen und mit welchen Strategien sie Naturkatastrophen bewältigen. Es fehlen zudem kurze Resümees, die erläutern, inwiefern aus dem geschichtswissenschaftlich dokumentierten Umgang mit Naturkatastrophen Lehren für die Gegenwart gezogen werden können und wie damit zur Lösung zukünftiger Probleme beigetragen werden kann.

Der Reiz für natur- und ingenieurwissenschaftlich orientierte Leser liegt eher im historisch reizvollen Detail der einzelnen Abhandlungen als in der Möglichkeit, für den heutigen Umgang mit Naturgefahren Erkenntnisse zu gewinnen und Rückschlüsse für ein integrales Risikomanagement zu ziehen. So ist das Buch für die gesamte Thematik rund um die (historischen) Naturgefahren eine ergiebige Quelle, es ist jedoch mehr ein etwas gar "akademisches" Lesebuch als ein tragfähiger Brückenschlag zur dringlich geforderten und für ein integrales Risikomanagement notwendigen Interdisziplinarität zwischen Sozial- und Geisteswissenschaften einerseits und Natur- und Ingenieurwissenschaften andererseits.

Walter J. Ammann Eidgenössisches Institut für Schneeund Lawinenforschung SLF, Davos

\title{
SOZIALE 圈TECHNIK ZEITSCHRIFT FÜR SOZIAL- UND UMWELTVERTRÄGLLCHE TECHNIKGESTALIUNG
} Mit Beitrögen zu:

\author{
$\square$ Technologie und Politik $\square$ Umwelt und Energie $\square$ Neve Biotechnologien \\ - Frauen und Technik $\square$ Informations- und Kommunikationstechnologien
} SOZIALE TECHNIK erscheint vierteljährlich. Ein Jahresabonnement kostet $€ 15$; (für Studierende $€ 10$,-)

Kostenloses Probeabo

und Abobestellungen:

Tel.: +43/316/81 39 09-0 / Fax: +43/316/81 0274

E-mail: waechter-@@ifz.tugraz.ot / Web: www.ifz.tugraz.ot/sote/ 